\title{
NEUTRON TRANSPORT IN EULERIAN COORDINATES WITH BULK MATERIAL MOTION
}

\author{
Randal S. Baker, Jon A. Dahl, and Erin J. Fichtl \\ Los Alamos National Laboratory \\ Computational Physics Group \\ rsb@lanl.gov; dahl@lanl.gov; efichtl@lanl.gov \\ Jim E. Morel \\ Department of Nuclear Engineering \\ Texas A\&M University \\ morel@tamu.edu \\ * - Corresponding author
}

\begin{abstract}
A consistent, numerically stable algorithm for the solution of the neutron transport equation in the presence of a moving material background is presented for one-dimensional spherical geometry. Manufactured solutions are used to demonstrate the correctness and stability of our numerical algorithm. The importance of including moving material corrections is shown for the r-process in proto-neutron stars.

Key Words: Neutron transport, material motion corrections, Eulerian coordinates, r-process
\end{abstract}

\section{INTRODUCTION}

The neutron transport equation is typically applied in situations where, other than temperatureinduced thermal motion, the background material is assumed to be at rest. However, it has also been long recognized that the assumption of no bulk material motion can lead to non-negligible errors in the neutron transport solution as material speeds start to approach neutron speeds, e.g. thermal neutron transport in coolant flow in a reactor (Perkins, 1970). Background (bulk) material motion has several potentially significant effects on neutron transport. The rate at which neutrons interact with the material is determined by the neutron speed relative to the material, so neutrons traveling with the direction of material flow undergo a reduced interaction rate, whereas those traveling against the direction of material flow experience an increased interaction rate. Neutron cross sections themselves are a function of the neutron energy as measured by the neutron speed relative to the background material, not the neutron speed in the lab frame. In addition, since neutron cross section data is evaluated with the target nuclei at rest, the actual kinematics of scattering and fission cross reactions must now be altered to take into account the direction of material flow.

For non-relativistic neutrons, where Galilean invariance is valid, there have been two historical approaches to dealing with the corrections necessary for transport against a moving material background. In the first approach, recognizing that neutron interaction rates are frame invariant, 
"effective" cross sections (and "effective" sources, if necessary) are formed using stationary cross section data scaled by the relative to lab frame velocities and a velocity Jacobian (Wienke et al., 1987a; Wienke and Hill, 1990; Wienke, 2005 ). While this approach allows the neutron transport solution to be performed within the lab frame, the resulting "effective" cross sections in this frame are now anisotropic for all reactions, including total removal and fission, with associated complications and potential instabilities in the transport solution.

The second approach transforms the solution of the transport equation to the co-moving frame, i.e., the reference frame where neutrons interact with the bulk material as if it were stationary (Wienke, 1974, 2005; Wienke et al., 1987b). This transformation results in a transport equation with a pseudo-acceleration term generated by material acceleration (spatial or temporal) and a material velocity effect term. The material velocity effect term in the "modified" Eulerian material coordinate system manifests itself as a convective Lagrangian derivative in the comoving frame (Wienke 1974). The total and fission cross sections remain isotropic in the comoving frame, however, and this is the frame in which we choose to pursue our transport solution. Unlike Wienke (1974) and Wienke et al. (1987b), we will implement our approach in a material coordinate system which is strictly Eulerian, there defining, developing and applying consistent, numerically stable solution techniques for both the material velocity and material acceleration terms.

\section{THE BOLTZMANN TRANSPORT EQUATION IN THE CO-MOVING FRAME}

Let $N(\vec{r}, \vec{q}, t)$ be the neutron density in the co-moving frame, where $\vec{r}$ is the spatial coordinate in the lab (Eulerian) frame, $\vec{q}$ is the co-moving frame neutron velocity defined by $\vec{q}(\vec{r}, t)=\vec{v}-$ $\vec{u}(\vec{r}, t)$, and $\vec{v}$ and $\vec{u}(\vec{r}, t)$ are the neutron and material velocities in the lab frame, respectively. Since the Boltzmann transport equation may be viewed simply as a statement of neutron conservation that is independent of the frame, we can then state that

$$
\begin{aligned}
\frac{d}{d t} N(\vec{r}, \vec{q}, t) & =\frac{\partial}{\partial t} N(\vec{r}, \vec{q}, t)+\frac{d \vec{r}}{d t} \cdot \frac{\partial}{\partial \vec{r}} N(\vec{r}, \vec{q}, t)+\frac{d \vec{q}}{d t} \cdot \frac{\partial}{\partial \vec{q}} N(\vec{r}, \vec{q}, t) \\
& =\dot{S}(\vec{r}, \vec{q}, t)-q(\vec{r}, t) \Sigma_{T}(\vec{r}, q, t) N(\vec{r}, \vec{q}, t)
\end{aligned}
$$

where $\dot{S}$ is the neutron gain rate due to scattering, fission, and external sources, and the neutron removal rate is $q \Sigma_{T} N$.

Recognizing that $d \vec{r} / d t=\vec{v}=\vec{q}+\vec{u}$ and, for neutrons, $d \vec{v} / d t=d \vec{q} / d t+d \vec{u} / d t=0$, we can then write Eq. (1), after dropping the variable indices for clarity, as

$$
\frac{\partial N}{\partial t}+(\vec{q}+\vec{u}) \cdot \nabla_{\vec{r}} N+q \Sigma_{T} N=\dot{S}+\nabla_{\vec{q}} N \cdot\left[\frac{\partial}{\partial t}+(\vec{q}+\vec{u}) \cdot \nabla_{\vec{r}}\right] \vec{u}
$$

Using the vector relationship $(\nabla a) \cdot \vec{b}=\nabla \cdot(a \vec{b})-a(\nabla \cdot \vec{b})$, and since $\nabla_{\vec{q}} \cdot \vec{u}=0$, we have $\nabla_{\vec{q}} \cdot\left(\vec{q} \cdot \nabla_{\vec{r}}\right) \vec{u}=\nabla_{\vec{r}} \cdot \vec{u}$. After some manipulation, we can rewrite Eq. (2) in conservative form as

$$
\frac{\partial N}{\partial t}+\nabla_{\vec{r}} \cdot(\vec{u} N)+\vec{q} \cdot \nabla_{\vec{r}} N+q \Sigma_{T} N=\dot{S}+\nabla_{\vec{q}} \cdot\left(N\left[\frac{\partial}{\partial t}+(\vec{q}+\vec{u}) \cdot \nabla_{\vec{r}}\right] \vec{u}\right)
$$


Here, the terms in black are the standard Boltzmann transport equation when the background material is stationary, the term in blue is a spatial advection term due to material velocity, and the term in red is an energy/angle (or neutron velocity) advection term due to material acceleration.

Although we have treated the neutron velocity in the co-moving frame as a dependent quantity, i.e., $\vec{q}(\vec{r}, t)=\vec{v}-\vec{u}(\vec{r}, t)$, and the neutron velocity $\vec{v}$ as independent, we will switch these roles during our numerical solution. That is, we will now consider $\vec{q}=\vec{v}(\vec{r}, t)-\vec{u}(\vec{r}, t)$ as an independent quantity, allowing us to reuse standard neutron transport algorithms developed for stationary background materials.

\section{DISCRETIZATION OF THE TRANSPORT EQUATION FOR ONE-DIMENSIONAL SPHERICAL GEOMETRIES IN THE CO-MOVING FRAME}

The analytic form of the Boltzmann transport equation for one-dimensional spherical geometries in the co-moving frame was first presented by Wienke for "modified" Eulerian coordinates (Wienke, 1974). It is straightforward to instead perform this derivation for the Eulerian comoving frame, where the material velocity is in the lab frame and the neutron transport is performed in the co-moving frame $\vec{q}$, so we do not show it here. The explicit representation of the transport equation in spherical geometry produces a somewhat more involved form of Eq. (3) since we must not only transform our spatial coordinate system from $\vec{r}=x \hat{\imath}+y \hat{\jmath}+z \hat{k}$ to that of $\vec{r}=r \hat{\imath}, r=\sqrt{x^{2}+y^{2}+z^{2}}$, but we must also transform our velocity coordinate system from $\vec{q}=q_{x} \hat{\imath}+q_{y} \hat{\jmath}+q_{z} \hat{k}$ to the standard transport spherical velocity coordinate system of $\vec{q}=q \vec{\Omega}$, $q=\sqrt{q_{x}^{2}+q_{y}^{2}+q_{z}^{2}}, \vec{\Omega}=\mu \hat{\imath}+\eta \hat{\jmath}+\xi \hat{k}$. The resulting analytic equation for spherical geometry with moving material corrections is

$$
\begin{aligned}
q \frac{\partial \Psi}{\partial t} & +\frac{q}{r^{2}} \frac{\partial}{\partial r}\left(r^{2} u \Psi\right)+q^{2}\left\{\frac{\mu}{r^{2}} \frac{\partial}{\partial r}\left(r^{2} \Psi\right)+\frac{1}{r} \frac{\partial}{\partial \mu}\left[\left(1-\mu^{2}\right) \Psi\right]\right\}+q^{2} \Sigma_{T} \Psi \\
& =q^{2} \dot{S}+\frac{\partial}{\partial q}\left[\left\{\mu q\left(\dot{u}+u \frac{\partial u}{\partial r}\right)+\mu^{2} q^{2} \frac{\partial u}{\partial r}+q^{2}\left(1-\mu^{2}\right) \frac{u}{r}\right\} \Psi\right] \\
& +\left(\dot{u}+u \frac{\partial u}{\partial r}\right) \frac{\partial}{\partial \mu}\left[\left(1-\mu^{2}\right) \Psi\right]+q\left[r \frac{\partial}{\partial r} \frac{u}{r}\right] \frac{\partial}{\partial \mu}\left[\mu\left(1-\mu^{2}\right) \Psi\right]
\end{aligned}
$$

where we have rewritten the equation in terms of the angular flux $\Psi(r, q, \mu, t)=q N(r, q, \mu, t)$. This equation must now be discretized in space, angle, and energy to enable a numerical solution.

We perform our discretization with the requirement that, for constant $N(r, q, \mu, t)$ but arbitrary material velocity $u(r, t)$, the spatial advection operator in blue on the left-hand side of Eq. (4) and the material advection operator in red on the right-hand side must identically cancel since, as can be seen from Eq. (3), these terms each reduce to $N\left(\nabla_{\vec{r}} \cdot \vec{u}\right)$. We also require that our discretization maintains backwards compatibility with existing multigroup $S_{N}$ transport implementations. Our approach for the spatial and angular $\left(S_{N}\right)$ discretizations is standard (Lewis and Miller, 1993). To perform our energy (speed) discretization for energy group $g$, with 
$\Delta q_{g}=q\left(E_{g-1}\right)-q\left(E_{g}\right)=q_{g p}-q_{g m}, g=1 \rightarrow G$, where we have retained the traditional ordering of energy groups, we recognize that the physical number of neutrons in a phase space element must be independent of whether a Cartesian or spherical velocity coordinate system is used, and also whether neutron speed is expressed as a function of $q$ or of energy $E$. Thus,

$$
N(\vec{r}, \vec{q}, t) d \vec{r} d \vec{q}=q^{2} N(\vec{r}, q, \vec{\Omega}, t) d \vec{r} d q d \vec{\Omega}=\frac{\Psi(\vec{r}, E, \vec{\Omega}, t)}{q} d \vec{r} d E d \vec{\Omega}
$$

Since $d E=m q d q$, where $m$ is the neutron mass, it follows that

$$
\Psi(\vec{r}, E, \vec{\Omega}, t)=\frac{q^{2}}{m} N(\vec{r}, q, \vec{\Omega}, t)
$$

Using Eqs. (5-6), the standard definition of the multigroup flux $\psi_{g}$ when expressed as a function of $q$ becomes

$$
\begin{gathered}
\psi_{g}(\vec{r}, \vec{\Omega}, t)=\int_{\Delta E_{g}} \Psi(\vec{r}, E, \vec{\Omega}, t) d E=\int_{\Delta q_{g}} \frac{q^{2}}{m} N(\vec{r}, q, \vec{\Omega}, t) m q d q \\
=\int_{\Delta q_{g}} q^{2} \Psi(\vec{r}, q, \vec{\Omega}, t) d q
\end{gathered}
$$

Upon applying our discretizations, the resulting transport equation for the angular flux in spatial cell $r_{i}$, where $\Delta r_{i}=r_{i+1 / 2}-r_{i-1 / 2}=r_{i p}-r_{i m}$, with spatial cell edge areas $A_{i m}$ and $A_{i p}$, spatial volume $V_{i}$, for angle $m, \Delta \mu_{m}=\mu_{m+1 / 2}-\mu_{m-1 / 2}=\mu_{m p}-\mu_{m m}$ with quadrature weight $w_{m}$, in energy group $g$, with material speeds $u_{i}, u_{i p}=u_{i+1 / 2}$ and $u_{i m}=u_{i-1 / 2}$, is

$$
\begin{gathered}
\left\langle\frac{1}{q}\right\rangle_{g} \frac{\partial \psi_{i, m, g}}{\partial t}+\left\langle\frac{1}{q}\right\rangle_{g}\left(\frac{A_{i p} u_{i p} \psi_{i p, m, g}-A_{i m} u_{i m} \psi_{i m, m, g}}{V_{i}}\right) \\
+\mu_{m}\left(\frac{A_{i p} \psi_{i p, m, g}-A_{i m} \psi_{i m, m, g}}{V_{i}}\right)+\left\langle\frac{1}{r}\right\rangle_{i} \frac{\alpha_{m p}^{\prime} \psi_{i, m p, g}-\alpha_{m m} \psi_{i, m m, g}}{w_{m}}+\Sigma_{T, i, g} \psi_{i, m, g} \\
=\dot{S}_{i, m, g}+\left\langle\frac{1}{q^{2}}\right\rangle_{g}\left\langle\dot{u}+u \frac{\partial u}{\partial r}\right\rangle_{i}\left(\frac{\alpha_{m p} \psi_{i, m p, g}-\alpha_{m m} \psi_{i, m m, g}}{w_{m}}\right) \\
+\left\langle\frac{1}{q}\right\rangle_{g}\left(\left\langle\frac{\partial u}{\partial r}\right\rangle_{i}-\left\langle\frac{u}{r}\right\rangle_{i}\right)\left(\frac{\zeta_{m p} \psi_{i, m p, g}-\zeta_{m m} \psi_{i, m m, g}}{w_{m}}\right) \\
+\mu_{m}\left\langle\dot{u}+u \frac{\partial u}{\partial r}\right\rangle_{i}\left(q_{g p} \Psi_{i, m, g p}-q_{g m} \Psi_{i, m, g m}\right) \\
+\mu_{m}^{2}\left(\left\langle\frac{\partial u}{\partial r}\right\rangle_{i}-\left\langle\frac{u}{r}\right\rangle_{i}\right)\left(q_{g p}^{2} \Psi_{i, m, g p}-q_{g m}^{2} \Psi_{i, m, g m}\right) \\
+\left\langle\frac{u}{r}\right\rangle_{i}\left(q_{g p}^{2} \Psi_{i, m, g p}-q_{g m}^{2} \Psi_{i, m, g m}\right)
\end{gathered}
$$

with the following definitions: 


$$
\begin{aligned}
& 4 \pi \int_{\Delta r_{i}} r^{2} \psi d r \equiv \psi_{i} V_{i} \\
& 4 \pi \int_{\Delta r_{i}} r \psi d r \equiv\left\langle\frac{1}{r}\right\rangle_{i} \psi_{i} V_{i}, \quad\left\langle\frac{1}{r}\right\rangle_{i}=\frac{A_{i p}-A_{i m}}{V_{i}} \\
& 4 \pi \int_{\Delta r_{i}} r u \psi d r \equiv\left\langle\frac{u}{r}\right\rangle_{i} \psi_{i} V_{i}, \quad\left\langle\frac{u}{r}\right\rangle_{i}=\frac{1}{2}\left(\frac{A_{i p} u_{i p}-A_{i m} u_{i m}}{V_{i}}-\left\langle\frac{\partial u}{\partial r}\right\rangle_{i}\right) \\
& 4 \pi \int_{\Delta r_{i}} r^{2}\left(\dot{u}+u \frac{\partial u}{\partial r}\right) \psi d r \equiv\left\langle\dot{u}+u \frac{\partial u}{\partial r}\right\rangle_{i} \psi_{i} V_{i}, \quad\left\langle\dot{u}+u \frac{\partial u}{\partial r}\right\rangle_{i}=\langle\dot{u}\rangle_{i}+\langle u\rangle_{i}\left\langle\frac{\partial u}{\partial r}\right\rangle_{i} \\
& 4 \pi \int_{\Delta r_{i}} r^{2} \frac{\partial u}{\partial r} \psi d r \equiv\left\langle\frac{\partial u}{\partial r}\right\rangle_{i} \psi_{i} V_{i}, \quad\left\langle\frac{\partial u}{\partial r}\right\rangle_{i}=\frac{u_{i p}-u_{i m}}{\Delta r_{i}} \\
& \int_{\Delta \mu_{m}} \psi d \mu \equiv w_{m} \psi_{m} \\
& \int_{\Delta \mu_{m}} \mu \psi d \mu \equiv w_{m} \mu_{m} \psi_{m} \\
& \int_{\Delta \mu_{m}} \mu^{2} \psi d \mu \equiv w_{m} \mu_{m}^{2} \psi_{m} \\
& \int_{\Delta \mu_{m}} \frac{\partial}{\partial \mu}\left[\left(1-\mu^{2}\right) \psi\right] d \mu \equiv \alpha_{m p} \psi_{m p}-\alpha_{m m} \psi_{m m}, \quad \alpha_{m p}-\alpha_{m m}=-2 \mu_{m} w_{m} \\
& \int_{\Delta \mu_{m}} \frac{\partial}{\partial \mu}\left[\left(1-\mu^{2}\right) \psi\right] d \mu \equiv \alpha_{m p}^{\prime} \psi_{m p}-\alpha_{m m}^{\prime} \psi_{m m}, \quad \alpha_{m p}^{\prime}-\alpha_{m m}^{\prime}=-\mu_{m} w_{m} \\
& \int_{\Delta \mu_{m}} \frac{\partial}{\partial \mu}\left[\mu\left(1-\mu^{2}\right) \psi\right] d \mu \equiv \zeta_{m p} \psi_{m p}-\zeta_{m m} \psi_{m m}, \quad \zeta_{m p}-\zeta_{m m}=\left(1-3 \mu_{m}^{2}\right) w_{m} \\
& \int_{\Delta q_{g}} q \Psi(\vec{r}, q, \vec{\Omega}, t) d q \equiv\left\langle\frac{1}{q}\right\rangle_{g} \psi_{g}
\end{aligned}
$$

and

$$
\int_{\Delta q_{g}} \Psi(\vec{r}, q, \vec{\Omega}, t) d q \equiv\left\langle\frac{1}{q^{2}}\right\rangle_{g} \psi_{g}
$$


In this form, as $\langle u / r\rangle_{i} \rightarrow 0$ and $\langle 1 / r\rangle_{i} \rightarrow 0$, we recover the equation for slab geometry (not shown here). Note that while the $\psi_{g}$ in Eq. (8) are now standard multigroup fluxes, the group edge values $\Psi_{g m}$ and $\Psi_{g p}$ instead represent point values for $q_{g+1 / 2} N\left(q_{g+1 / 2}\right)$ and $q_{g-1 / 2} N\left(q_{g-1 / 2}\right)$, respectively. Also, in Eq. (8d), $\langle u\rangle_{i}$ and $\langle\dot{u}\rangle_{i}$ are evaluated at the cell centroid, and to complete Eqs. (8d) and (8e) we have assumed $\vec{u}$ is linear in $(r, t)$.

For conservation, the material acceleration terms in red on the right-hand side must integrate to zero when summed over all angles $m$ and energy groups $g$. Upon performing this summation, recognizing that $\Psi_{i, m,(g+1)}=\Psi_{i, m, g p}$ and $\psi_{i,(m+1), g}=\psi_{i, m p, g}$ and eliminating terms that cancel, we obtain

and

$$
\begin{gathered}
\Psi_{i, m, 1 / 2}=\Psi_{i, m, G+1 / 2}=0 \\
\alpha_{1 / 2}=\alpha_{M+1 / 2}=0
\end{gathered}
$$

$$
\zeta_{1 / 2}=\zeta_{M+1 / 2}=0
$$

Here, $M$ is the total number of angles in the quadrature set, e.g., six for $\mathrm{S}_{6}$, and $G$ is the total number of energy groups.

\section{STABILITY REQUIREMENTS FOR MOVING MATERIAL CORRECTIONS}

Direct incorporation of the additional spatial/angle/energy advection terms from Eq. (8) into a transport solver using standard solution techniques is problematic since they may, depending upon the material velocity, render the transport solver numerically instable. Instead, we choose to use operator splitting to handle the additional terms introduced by moving material corrections. We rewrite Eq. (8) as

where

$$
\left\langle\frac{1}{q}\right\rangle_{g} \frac{\partial \psi_{i, m, g}}{\partial t}+A \psi+B \psi-C \psi=\dot{S}_{i, m, g}
$$

$$
\begin{gathered}
A \psi \\
=\mu_{m}\left(\frac{A_{i p} \psi_{i p, m, g}-A_{i m} \psi_{i m, m, g}}{V_{i}}\right)+\left\langle\frac{1}{r}\right\rangle_{i} \frac{\alpha_{m p}^{\prime} \psi_{i, m p, g}-\alpha_{m m}^{\prime} \psi_{i, m m, g}}{w_{m}} \\
+\Sigma_{T, i, g} \psi_{i, m, g}
\end{gathered}
$$




$$
\begin{gathered}
=\left\langle\frac{1}{q^{2}}\right\rangle_{g}\left\langle\dot{u}+u \frac{\partial u}{\partial r}\right\rangle_{i}\left(\frac{\alpha_{m p} \psi_{i, m p, g}-\alpha_{m m} \psi_{i, m m, g}}{w_{m}}\right) \\
+\left\langle\frac{1}{q}\right\rangle_{g}\left(\left\langle\frac{\partial u}{\partial r}\right\rangle_{i}-\left\langle\frac{u}{r}\right\rangle_{i}\right)\left(\frac{\zeta_{m p} \psi_{i, m p, g}-\zeta_{m m} \psi_{i, m m, g}}{w_{m}}\right)+\mu_{m}\left\langle\dot{u}+u \frac{\partial u}{\partial r}\right\rangle_{i}\left(q_{g p} \Psi_{i, m, g p}\right. \\
\left.-q_{g m} \Psi_{i, m, g m}\right)+\mu_{m}^{2}\left(\left\langle\frac{\partial u}{\partial r}\right\rangle_{i}-\left\langle\frac{u}{r}\right\rangle_{i}\right)\left(q_{g p}^{2} \Psi_{i, m, g p}-q_{g m}^{2} \Psi_{i, m, g m}\right) \\
+\left\langle\frac{u}{r}\right\rangle_{i}\left(q_{g p}^{2} \Psi_{i, m, g p}-q_{g m}^{2} \Psi_{i, m, g m}\right)
\end{gathered}
$$

Given a time step over $t^{n+1 / 2}=t^{n-1 / 2}+\Delta t_{n}$, and with the colors denoting split levels, we then solve Eq. (10) as follows

$$
\begin{aligned}
& \left\langle\frac{1}{q}\right\rangle_{g} \frac{\psi_{i, m, g}^{n+1 / 2}-\psi_{i, m, g}^{n-1 / 2}}{\Delta t_{n}}+A \psi^{n}=\dot{S}_{i, m, g}^{n} \\
& \left\langle\frac{1}{q}\right\rangle_{g} \frac{\psi_{i, m, g}^{n+1 / 2}-\psi_{i, m, g}^{n+1 / 2}}{\Delta t_{n}}+B \psi^{n+1 / 2}=0 \\
& \left\langle\frac{1}{q}\right\rangle_{g} \frac{\psi_{i, m, g}^{n+1 / 2}-\psi_{i, m, g}^{n+1 / 2}}{\Delta t_{n}}-C \psi^{n+1 / 2}=0
\end{aligned}
$$

Eq. (14) represents the standard discrete ordinates $\left(S_{n}\right)$ transport equation with no material motion, and may now be solved using standard techniques (in our case, transport sweeps with diamond difference in space and angle, multigroup in energy, and Crank-Nicholson in time).

Eq. (15) is the spatial advection term due to material velocity, which we choose to solve explicitly in time, or

$$
\psi_{i, m, g}^{n+1 / 2}=\psi_{i, m, g}^{n+1 / 2}+\frac{\Delta t_{n}}{V_{i}}\left(A_{i m} u_{i m} \psi_{i m, m, g}^{n+1 / 2}-A_{i p} u_{i p} \psi_{i p, m, g}^{n+1 / 2}\right)
$$

For simplicity and robustness we use upwind differencing in space for this term, with the celledge angular flux defined in terms of its upwind cell-centered angular flux. Since we are solving the spatial advection term explicitly in time, we have now introduced a Courant-like condition for positivity/stability that limits the time step size such that the material velocity can "drag" particles no more than one spatial cell per time step.

Attempting to also solve the material acceleration term of Eq. (16) explicitly in time would result in an overly restrictive time step limit that would allow particles to be advected no more than one phase space cell in energy/angle per time step. Instead, Eq. (16) receives an implicit time treatment, or 


$$
\psi_{i, m, g}^{n+1 / 2}=\left(I-\frac{\Delta t_{n}}{\langle 1 / q\rangle_{g}} C\right)^{-1} \psi_{i, m, g}^{n+1 / 2}=M^{-1} \psi_{i, m, g}^{n+1 / 2}
$$

When upwinding in energy we must represent the group edge values $\Psi_{g m}$ and $\Psi_{g p}$ in terms of the group fluxes $\psi_{g}$. This is most straightforwardly done by recognizing that the average neutron density within a group $g$ is just the total number of neutrons within that group divided by the "volume" in $q$ for that group, or

$$
N_{g} \equiv \frac{\langle 1 / q\rangle_{g} \psi_{g}}{\int_{\Delta q_{g}} q^{2} d q}=\frac{\langle 1 / q\rangle_{g} \psi_{g}}{\left(q_{g p}^{3}-q_{g p}^{3}\right) / 3}=\frac{\langle 1 / q\rangle_{g} \psi_{g}}{V_{g}}
$$

We then assume that, depending upon the upwind direction, the point values for $\Psi_{g m}$ and $\Psi_{g p}$ may instead be approximated by $\Psi_{g m} \approx q_{g+1 / 2}\langle 1 / q\rangle_{g+1} \psi_{g+1} / V_{g+1}$ and $\Psi_{g p} \approx q_{g-1 / 2}\langle 1 /$ $q\rangle_{g} \psi_{g} / V_{g}$, or $\Psi_{g m} \approx q_{g+1 / 2}\langle 1 / q\rangle_{g} \psi_{g} / V_{g}$ and $\Psi_{g p} \approx q_{g-1 / 2}\langle 1 / q\rangle_{g-1} \psi_{g-1} / V_{g-1}$. This approximation is exact for constant $N$. The appropriate use of upwinding with Eq. (18) results in a matrix $M$ that, although not necessarily diagonally dominant, does have positive diagonal and non-positive off-diagonal elements.

The solution of the Boltzmann transport equation along the starting direction $\mu=-1$ is often used to provide the initial condition $\psi_{i, 1 / 2, g}$ needed for angular differencing schemes in curvilinear geometries (Lewis and Miller, 1993). Consider now a neutron headed inwards toward the sphere relative to a background material that is also accelerating inwards. As the material speed increases, the neutron maintains its direction of $\mu=-1$ in the co-moving frame, but its speed decreases until it is placed within the bottom energy group. At that point, in the spherical velocity coordinate system, our energy/angle advection operator must instantaneously transfer the neutron from $\mu=-1$ to $=+1$. This presents itself as a first-order discontinuity in the equation for the starting direction, resulting in a numerical discretization that cannot be closed. To avoid this difficulty we replace the use of starting directions with "step-start" in angle.

\section{VERIFICATION USING MANUFACTURED SOLUTIONS}

No known analytical solutions for the transport equation with moving material corrections exist, so we instead use the method of manufactured solutions to verify our numerical algorithms are correct. We assume a homogeneous, infinite (in both space and energy) sphere with $\Sigma_{T}(r, q, t)=$ 1 and no scattering or fission, i.e., purely absorbing, so that $\dot{S}$ is due only to external sources. We also assume a linear material velocity $u(r, t)=a+b r+c t$, where $a, b$, and $c$ are arbitrary constants.

To define our manufactured solution for the neutron density $N$ we choose to use a polynomial representation of arbitrary order $n$ in speed that is isotropic in angle, uniform in space, and constant in time, i.e., $N(r, q, \mu, t)=q^{n}, n \geq 0$, or $\Psi(r, q, \mu, t)=q^{n+1}$. Using these definitions for $u$ and $\psi$ in Eq. (4), then integrating the resulting analytic source over the spatial cell $\Delta r_{i}$ and energy group $g$, produces the multigroup source 


$$
\begin{gathered}
\dot{S}_{i, g}(\mu, t) \\
=\left[\frac{\left(q_{g p}^{n+4}-q_{g m}^{n+4}\right)}{n+4}-n \frac{1}{3}\left(b+2\left\langle\frac{u}{r}\right\rangle_{i}\right) \frac{\left(q_{g p}^{n+3}-q_{g m}^{n+3}\right)}{n+3}\right] P_{0}(\mu) \\
-n\left\langle\dot{u}+u \frac{\partial u}{\partial r}\right\rangle_{i} \frac{\left(q_{g p}^{n+2}-q_{g m}^{n+2}\right)}{n+2} P_{1}(\mu)-n \frac{2}{3}\left(b-\left\langle\frac{u}{r}\right\rangle_{i}\right) \frac{\left(q_{g p}^{n+3}-q_{g m}^{n+3}\right)}{n+3} P_{2}(\mu)
\end{gathered}
$$

for the associated solution $\psi_{i, g}(\mu, t)=\left(q_{g p}^{n+4}-q_{g m}^{n+4}\right) /(n+4)$.

Since our manufactured solution was postulated in an infinite medium in space and energy, we modify the right spatial and top energy boundary conditions to advect in the neutron flux specified by the associated solution. After doing so, since our discretization was based upon preserving the constant solution, we expect and obtain essentially machine accuracy for manufactured solutions when $n=0$, irrespective of the magnitude of the material velocity or acceleration. For $n>0$, there is a discretization error whose magnitude decreases as the number of energy groups and size of the quadrature set are increased. Shown below in Fig. 1 is the $L_{2}$ error norm in the angular flux for $n=1\left(\psi=q^{2}\right)$. The test problem consists of a sphere with radius 4.0 and eight spatial cells, a maximum neutron speed of $q_{1 / 2}=1.0$, uniformly divided into $G$ energy groups, an initial condition equal to the exact solution randomly perturbed by $\pm 5 \%$, a material velocity $u=-1+r+t$, and a constant time step of 0.01 . Calculations were performed using moving material corrections with $S_{2} / G=2, S_{4} / G=4, S_{8} / G=8$, as well as $S_{8} / G=8$ and no moving material corrections. Although a rigorous order of convergence study has not yet been performed, the magnitude of the error norm is monotonically decreasing as the quadrature and group structures are refined.

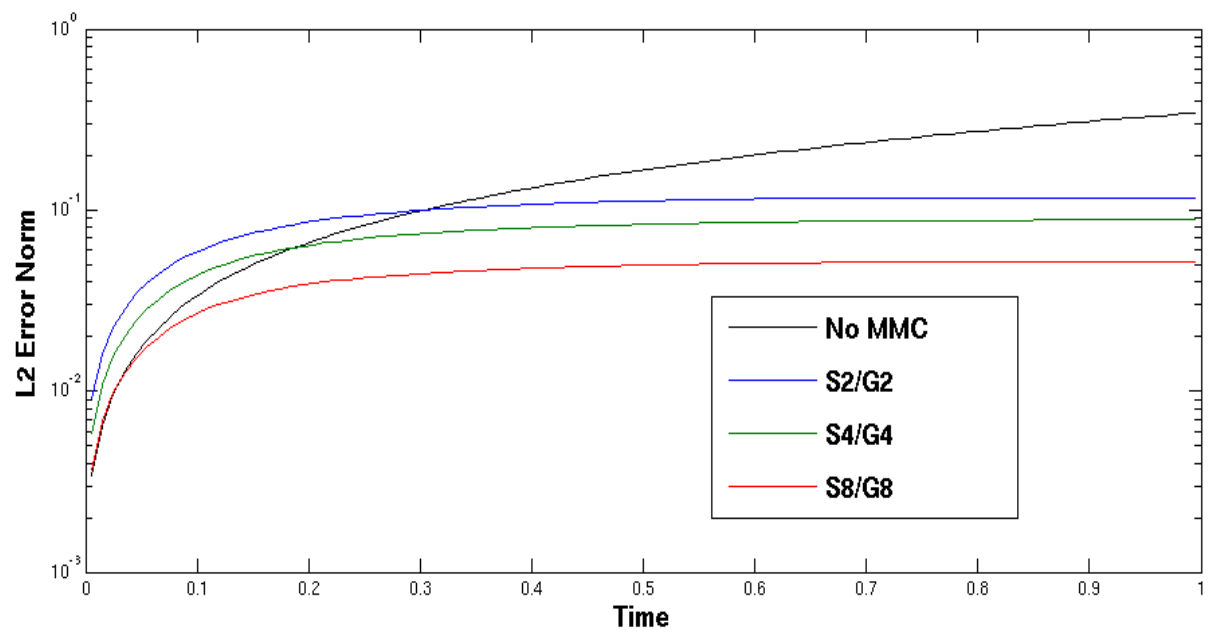

Figure 1. $\mathrm{L}_{2}$ Error Norm for $n=1$

\section{APPLICATION TO NUCLEAR ASTROPHYSICS}

Core-collapse supernovae are powered by the collapse of a stellar core into either a neutron star or a black hole. The potential energy released in this collapse powers an explosion that disrupts 
the star. As this explosive shock drives through the star, some material falls back onto the newly formed neutron star, creating secondary outbursts. Fryer et al. (2006) argued that these outbursts could be the site of r-process nucleosynthesis, i.e., the formation of elements through the rapid capture of neutrons onto heavy seed nuclei. Astronomers believe that this rapid neutron capture process is responsible for the creation of approximately one half of the atomic nuclei in the universe that are heavier than iron. In this fallback r-process scenario, heavy "seed" nuclei (e.g., iron) previously formed in the stellar process undergo a series of rapid neutron captures as they fall back toward the neutron star, and are then swept up in the outflow.

The standard assumption used in calculating these capture rates within the astrophysics community is that the neutron spectrum is thermal, i.e., Maxwellian, due to the extreme densities and associated collision rates encountered during the r-process. However, the gravitational fields and turbulent flows encountered in a proto-neutron star generate large material velocities and corresponding accelerations, which should be accounted for in any neutron transport calculation used to model this process as they may alter the neutron spectrum.

Consider a slab geometry with vacuum boundary conditions, where the left half consists of He with a density of $10^{4} \mathrm{gm} / \mathrm{cm}^{3}$ at a temperature of $100 \mathrm{keV}$, the right half of $\mathrm{Ni}$ with a density of $2.5 \times 10^{4} \mathrm{gm} / \mathrm{cm}^{3}$ at a temperature of $10 \mathrm{keV}$, and an isotropic neutron source incident on the left boundary with a $100 \mathrm{keV}$ Maxwellian spectrum. This geometry is meant to be representative of a $\mathrm{Ni}$ seed pellet falling back into the proto-neutron star. Neglecting moving material corrections, solution of this problem results in a neutron flux that is Maxwellian in shape at $100 \mathrm{keV}$ within the He region, near-Maxwellian (with some perturbations due to absorption) at $10 \mathrm{keV}$ in the $\mathrm{Ni}$ layer immediately adjacent to the He (shown below by the red line in Fig. 2), then rapidly diminishing in magnitude as we move further into the Ni region. The r-process is dominated by neutron captures immediately adjacent to the He-Ni interface because of the material densities involved and the resulting opaqueness of the Ni region.

Now consider the same problem geometry, but where the material in the He region has a leftgoing velocity of $0.001 \mathrm{~cm} / \mathrm{sh}$ (one shake is $10^{-8}$ seconds), and the material in the Ni region has a left-going velocity of $5.001 \mathrm{~cm} / \mathrm{sh}$. Although both material regions are traveling in the same direction, the difference in velocities presents itself as a strong spatial material acceleration to neutrons crossing between the two regions. This material acceleration at the He-Ni interface produces an effective increase in energy for neutrons traveling from the He region into the $\mathrm{Ni}$, resulting in a neutron spectrum immediately inside the Ni that is significantly non-Maxwellian (shown below by the blue line in Fig. 2). Although not shown here, the moving material correction also results in somewhat increased penetration by neutrons into the Ni interior. This is most likely due to the forward-directed $(\mu \rightarrow+1)$ angular shift from spatial acceleration for neutrons entering the $\mathrm{Ni}$ region.

More detailed analysis will be required to evaluate the effects of the spectral shift from moving material corrections on the r-process and neutron captures in proto-neutron stars. It should also be noted that, while the co-moving frame is the correct frame within which to calculate materialinteraction rates such as neutron capture, transformation back to the lab frame would be required for observables in the lab frame. 


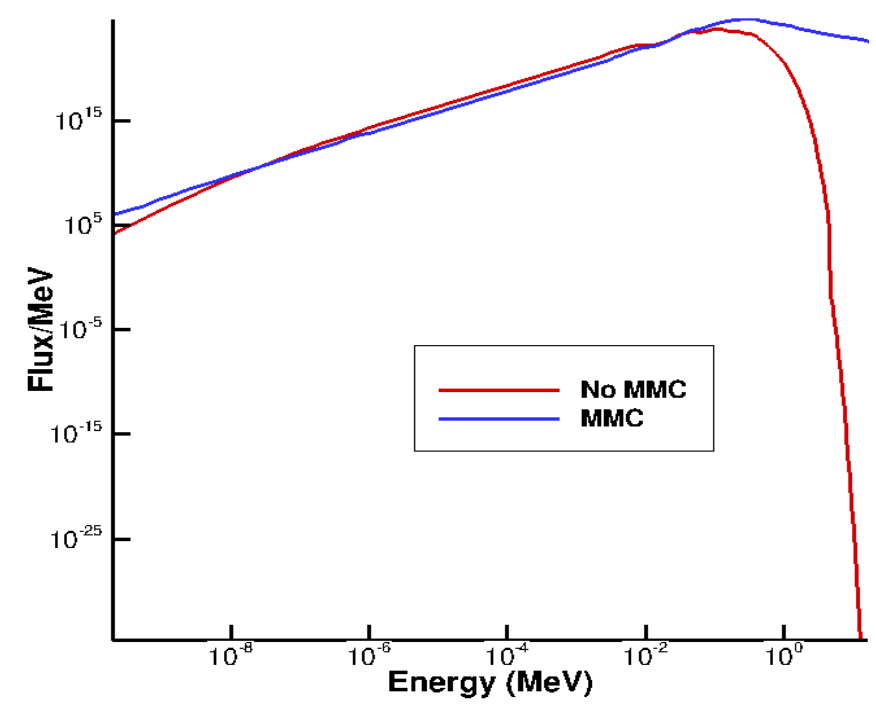

Figure 2. Spectral Shift with Moving Material Corrections

\section{CONCLUSIONS AND FUTURE WORK}

A consistent, stable numerical algorithm for the solution of the neutron transport equation in the presence of a moving material background has been presented for one-dimensional spherical geometries. Manufactured solutions have been used to verify the correctness and stability of our numerical algorithm, and the potential importance of accounting for the effects of moving material corrections in applications where material speeds start to approach neutron speeds has been demonstrated. Such application areas may perhaps be more widespread than realized, and hopefully our outline of a practical algorithm for determining the effects of moving material corrections within them will lead to their being included in future computations.

While extension of our existing algorithm to multi-dimensions would be straightforward, potential complications when material velocities align with quadrature directions leads us to choose a different path. Although this alignment difficulty can only occur along a starting direction in one- and two-dimensional geometries, it could potentially occur in any direction in three-dimensional geometries. To avoid the difficulties created by this alignment as discussed above in Section 4, we will instead replace our integrals over cell velocity volumes with surface integrals by applying the divergence theorem. We will also present a more accurate upwinding scheme for determining the group edge values $\Psi_{g m}$ and $\Psi_{g p}$. This work will be reported on in a future article.

\section{ACKNOWLEDGMENTS}

We thank Bruce Wienke for his many helpful insights, encouragement, and suggestions on tackling this issue, and Chris Fryer for guiding us on our investigation into neutron proto-stars. 
Los Alamos National Laboratory is operated by Los Alamos National Security, LLC, for the United States Department of Energy under Contract No. DE-AC52-06NA25396.

\section{REFERENCES}

Fryer, C.L., Herwig, F., Hungerford, A., Timmes, F.X., 2006. Supernova Fallback: A Possible Site for the r-Process. Astrophysical Journal. 646, L131-L134.

Lewis, E.E., Miller, Jr., W.F., 1993. Computational Methods of Neutron Transport, first ed. American Nuclear Society, La Grange Park, Illinois.

Perkins, S.T., 1970. Doppler Broadening in a Medium with Both Temperature and Boundary Motion. Nucl. Sci. Eng. 39, 25-31.

Wienke, B.R., 1974. Transport Equation in Modified Eulerian Coordinates. Phy. Fluids. 17, $1135-1138$.

Wienke, B.R., Hill, T.R., Whalen, P.P., 1987a. Multigroup Particle Transport in a Moving Material. J. Comp. Phy. 72, 177-201.

Wienke, B.R, Hill, T.R, Whalen, P.P, 1987b. Eulerian and Lagrangian Particle Transport with Drag. Computer Phy. Comm. 43, 171-180.

Wienke, B.R, Hill, T.R., 1990. Effective Cross Sections and Sources in Moving Material. Nucl. Sci. Eng. 104, 188-196.

Wienke, B.R., 2005. Transport Equations in Moving Material, Part I: Neutrons and Photons. Prog. Nucl. Eng. 46, 13-55. 